\title{
Multilateralismus auf dem Rückzug? Chancen und Herausforderungen der liberalen Weltordnung. Ein Bericht zum Heidelberger Dialog zur internationalen Sicherheit im November 2019
}

\author{
Marie Müller • Lukas Hassebrauck • Charlotte Liebrecht • \\ Hannah Schmidt
}

Online publiziert: 17. Juni 2020

(C) Der/die Autor(en) 2020

\section{Einleitung}

Migrationskrise, Brexit, Klimawandel - die Rede von den aktuellen Krisenerscheinungen des Multilateralismus ist allgegenwärtig. Leicht könnte man den Eindruck gewinnen, dass die westlichen Demokratien ihre Unterstützung für normgeleitetes und kooperatives Handeln in der internationalen Politik aufgegeben haben und sich in ein Zeitalter des Skeptizismus gegenüber Formen der multilateralen Zusammenarbeit begeben. Der Rückzug demokratischer Staaten aus Foren der Global Governance, wie dem Menschenrechtsrat der Vereinten Nationen oder dem Klimaabkommen von Paris, sind dabei nur zwei der unzähligen Beispiele dieser Tendenz. In den Mittelpunkt der Debatte rückt eine zentrale Frage: Gibt es eine Renaissance unilateralen Handelns in der internationalen Politik?

Doch so einseitig scheint es nicht zu sein: Denn während der Aufbau und der Einsatz in und für multilaterale Institutionen nach dem Zweiten Weltkrieg zunächst das Spielfeld der westlichen und demokratischen Staaten war, hat sich die Situation in den vergangenen Jahren gewandelt. Mehr und mehr treten autokratische Staaten auf das Parkett der internationalen Zusammenarbeit und bieten sich bereitwillig als Vorreiter neuer multilateraler Institutionen an, wie bspw. in der Asiatischen

\footnotetext{
M. Müller $(\bowtie) \cdot$ L. Hassebrauck $\cdot$ C. Liebrecht $\cdot$ H. Schmidt Institut für Politische Wissenschaft, Ruprecht-Karls-Universität Heidelberg, Forum für internationale Sicherheit Heidelberg e.V., Bergheimer Straße 58, 69115 Heidelberg, Deutschland

E-Mail: mueller@fis-hd.de

L. Hassebrauck

E-Mail: hassebrauck@ fis-hd.de

C. Liebrecht

E-Mail: liebrecht@fis-hd.de

H. Schmidt

E-Mail: schmidt@fis-hd.de
} 
Infrastrukturinvestmentbank. Gleichzeitig streben sie aber auch nach mehr Einfluss in der Agendasetzung etablierter Organisationsstrukturen und verbünden sich zu gemeinsamen Positionen. Was bedeutet dieser neue autokratische Multilateralismus für die Ausgestaltung der zukünftigen internationalen Ordnung und Organisationen? Und welche Konsequenzen ergeben sich aus diesen Entwicklungen für das System multilateraler Zusammenarbeit?

Zum elften Mal lud das Forum für internationale Sicherheit Heidelberg vor diesem Hintergrund vom 22. bis 23. November 2019 zum Heidelberger Dialog zur internationalen Sicherheit. Unter dem Titel Multilateralismus auf dem Rückzug? Chancen und Herausforderungen der liberalen Weltordnung diskutierten Teilnehmer*innen und Dozent*innen aus ganz Deutschland über mehrere Tage aus interdisziplinärer Perspektive heraus die verschiedensten Aspekte des Themenkomplexes und entwarfen während der Fachtagung mögliche Handlungsoptionen.

\section{Multilateralismus und das Welthandelssystem - Chancen, Risiken, Herausforderungen}

Den Auftakt des Heidelberger Dialogs bildete ein Impulsvortrag von Prof. Dr. Achim Wambach (Leibnitz-Zentrum für Europäische Wirtschaftsforschung Mannheim) mit dem Thema America first, Made in China 2025 - Und Europa?, der aus ökonomischer Sichtweise die drei größten Wirtschaftsregionen der Welt in den Bereichen Handel und Klima beleuchtete.

Zunächst stellte Prof. Wambach die Folgen der US-Wirtschaftspolitik unter Präsident Donald Trump heraus, nach der bestehende Freihandelsabkommen mit China und Europa in den Hintergrund rückten. Trump erschwere durch seine Politik eine kooperative Zusammenarbeit der ehemaligen engen Partner: In Europa führe das US-amerikanische Steuergesetz, nach dem Unternehmen nur in einem Land Steuern zahlen müssen, zu einem harten Wettbewerb um Firmenstandorte. Durch den Wirtschaftskrieg und einen verstärkten Protektionismus würde zudem versucht, chinesische Güter durch hohe Zölle unattraktiv für US-Bürger*innen zu machen.

Am Ende dieses Handelsstreits könnten die USA nach Prof. Wambach aber als Verlierer dastehen, sei Chinas Anteil am Welthandel in den letzten 15 Jahren doch auf $19 \%$ gewachsen. China verfolge vor allem zwei Wirtschaftsstrategien: das Infrastrukturprojekt der sogenannten neuen Seidenstraße (One Belt, One Road Initiative), mit dem Handelswege zwischen Asien, Afrika und Europa ausgebaut werden, und das Projekt Made in China 2025, mit dem ausländische Technologie vor allem im Bereich der künstlichen Intelligenz und des Deep Learnings durch chinesische ersetzt wird. Länder wie Deutschland hätten sich das Aufstreben der Volksrepublik aber durchaus zunutze gemacht und insbesondere in Bereichen der Chemie- und Pharmaindustrie, aber auch im Maschinen- und Autobau von dieser Entwicklung profitiert.

Mit Blick auf die wirtschaftliche Zukunft des europäischen Kontinents prophezeite Prof. Wambach infolge des bevorstehenden Austritts Großbritanniens aus der Europäischen Union einen erheblichen Einbruch der Wirtschaftskraft. Der Verlust eines der wirtschaftlich stärksten Mitgliedstaaten würde aber nicht nur den Europä- 
ischen Wirtschaftsraum in Mitleidenschaft ziehen. Vielmehr habe der Brexit auch einen direkten Einfluss auf die zukünftige internationale Verhandlungsposition der EU. Das wiederum könne den internationalen Trend von multi- zu bilateralen Handelsabkommen weiter befeuern.

In der Klimapolitik sprach sich Prof. Wambach für mehr Engagement der drei großen Weltwirtschaften aus. Kein anderes Politikfeld sei so sehr von einem funktionierenden Multilateralismus abhängig. Entsprechend müssten die USA, die EU und China auch die gemeinsame Forschung und Entwicklung stärker vorantreiben und sich aktiver für Überlegungen wie $\mathrm{CO}^{2}$-Bepreisung einsetzen. Auch müsse weiter an der konkreten Umsetzung des Pariser Klimaabkommens gearbeitet werden, stünden doch genaue Handlungsanweisungen bisher aus. Abhilfe könnte nach Prof. Wambach hierbei die Nutzung etablierter Wirtschaftsmechanismen leisten. Eine Möglichkeit könnte demnach sein, Klimaschutz auch finanziell für Unternehmen attraktiv zu machen, indem ihnen für ihren Einsatz Vergünstigungen in anderen Bereichen oder gar Zuschüsse zugestanden würden. Ebenfalls sprach sich Prof. Wambach für eine Verschärfung von Strafmechanismen bei Verstößen von vereinbarten Abkommen aus und führte als technische Möglichkeit wirtschaftliche Repressionen an. Klimaschutz könne man, so Prof. Wambach, nur mit Reziprozität begegnen.

\section{Keynote: Make our planet great again? Internationale Klimapolitik zwischen Multilateralismus und Populismus}

Eine Keynote von Dr. Maximilian Jungmann (Heidelberg Center for the Environment und Momentum Novum) zum Thema Make our planet great again? Internationale Klimapolitik zwischen Multilateralismus und Populismus, die sich in zwei inhaltliche Teile gliederte, bildete den Auftakt für die sich anschließenden Workshops am zweiten Tag der Konferenz.

Dr. Jungmanns Vortrag begann mit einer Einführung in die Bedeutung des Multilateralismus für die internationale Klimapolitik, bei der er den inhaltlichen Schwerpunkt auf eine Definition und Erläuterung des Klimawandels sowie auf die Unterscheidung von Nachhaltigkeit und Umwelt legte. Anschließend gab Dr. Jungmann einen Überblick über den aktuellen Forschungsstand und erläuterte Implikationen des Klimawandels, vor allem in den Bereichen Gesundheit, globale Migration und der internationalen Sicherheit im Allgemeinen. Er betonte die Notwendigkeit, dass die internationale Staatengemeinschaft dringend Maßnahmen ergreifen müsste, um diese globalen Herausforderungen bewältigen zu können und verwies hierbei auf die Bedeutung der UN als internationales Forum der Kooperation.

Im zweiten Teil definierte der Referent die Begriffe Multilateralismus, Bilateralismus, Unilateralismus sowie Populismus und grenzte diese voneinander ab. Zudem erklärte er den Unterschied zwischen demokratischen und autokratischen Zugängen in der internationalen (Klima-)Politik. Dr. Jungmann beschrieb außerdem die Herausforderungen, denen sich Regime der internationalen Klimapolitik in den letzten Jahren gegenübersahen und verwies in diesem Kontext insbesondere auf den Rückzug der USA aus dem Pariser Klimaabkommen. Die Auswirkungen des Verlustes der US-amerikanischen Hegemonialstellung seien dabei nicht zwangsläufig 
negativ, sondern könnten auch multilaterale Reaktionen anderer, sonst defensiver Staaten hervorrufen. Nach Dr. Jungmann würde sich der Multilateralismus damit nicht zwangsläufig in einer Krise befinden, sondern grenzüberschreitenden und themenspezifischen Herausforderungen, veränderten thematischen Allianzen und neuen internationalen Führungsrollen gegenüberstehen.

Im Rahmen der anschließenden Diskussion sprachen die Teilnehmer*innen über die Bedeutung von Sanktionen, um die in den Klimaabkommen niedergeschriebenen Ziele zu erreichen. Im Mittelpunkt der Diskussion stand außerdem das Verhältnis zwischen internationaler, nationaler und föderaler Ebene bei Klimaverhandlungen.

\section{Workshop I: Crumbling from within? Der Rückzug demokratischer Staaten aus multilateralen Foren}

Unter der Leitung von Dr. Johannes Muntschick (Johannes-Gutenberg-Universität Mainz) widmete sich der erste Workshop dem Thema Crumbling from within? Der Rückzug demokratischer Staaten aus multilateralen Foren. Die Zielsetzung des Workshops bestand darin, anhand empirischer Fallbeispiele Antworten auf die Frage zu entwickeln, aus welchen Gründen sich Staaten seit einigen Jahren vermehrt aus globalen Einrichtungen zurückziehen und internationale Abkommen und Verträge aufkündigen.

Zu diesem Zweck erarbeiteten sich die Teilnehmer*innen zunächst die wesentlichen Charakteristika der liberalen Weltordnung, wie sie sich seit 1945 unter Führung der USA herausgebildet hatte. Den Kern dieser Ordnung bildeten ihrer Ansicht nach die Institutionalisierung eines Normgefüges mit universellem Geltungsanspruch sowie eine fortgeschrittene Verrechtlichung der zwischenstaatlichen Beziehungen. In ihrem Zentrum stünden internationale Organisationen wie die UN, die Welthandelsorganisation und der Nordatlantikpakt. Insbesondere seit Anfang der 1990er Jahre habe sich das Ordnungsmodell im Zuge verstärkter transnationaler Verflechtung und der ökonomischen Globalisierung auf seinem Höhepunkt befunden. Ab dem Jahr 2010 konnten die Teilnehmer*innen einen empirischen Rückgang bi- und multilateraler Abkommen verzeichnen.

Zur Identifizierung von Motiven für die Re-Unilateralisierung des außenpolitischen Verhaltens demokratischer Staaten wurden im Anschluss daran in Gruppen vier Fallbeispiele bearbeitet, die stellvertretend einzelne Ursachen illustrieren sollten. Unter den ausgewählten Fällen befanden sich die Neuverhandlung des nordamerikanischen Freihandelsabkommens durch die USA, der Rückzug der USA aus dem Klimaabkommen von Paris, der Austritt Großbritanniens aus der EU und der Austritt Japans aus der internationalen Wahlfangkommission.

Allgemein beobachteten die Teilnehmer*innen dabei eine Diskursverschiebung weg von den zahlreichen Vorteilen multilateraler Kooperation, wie bspw. der Verhaltenserwartbarkeit oder sinkende Interaktionskosten. Mit Blick auf die Güter Frieden und Sicherheit, aber auch Klima- und Naturschutz würde an ihre Stelle zunehmend die Problematisierung von Risiken, wie dem Trittbrettfahren, der Frage nach der relativen Verteilung von Nutzen, Kosten und Lasten oder dem Kollektivgutdilemma treten. 
Neben kulturellen Variablen konnten die Teilnehmer*innen nationale Interessen, kurzfristige elektorale Erwägungen, innenpolitische Determinanten, das Streben nach relativen Gewinnen und den populistischen Politikstil einzelner Staats- und Regierungschefs als ausschlaggebende Ursachen für die aktuelle Krise des Multilateralismus identifizieren. Ebenfalls konnte in einzelnen Fällen der anachronistische Charakter und die Verzerrung der aktuellen Weltordnung zugunsten westlicher Prinzipien und zulasten alternativer Ordnungsvorstellungen nachgewiesen werden. Die herrschende Legitimationskrise internationaler Institutionen lasse sich nach Meinung der Teilnehmer*innen nur durch eine schärfere Sanktionierung nonkonformen Verhaltens lösen. Neben naming, blaming, and shaming seien zunehmend auch wirksamere Maßnahmen auf der Ebene der internationalen Politik gefragt.

\section{Workshop II: Autokratien und Multilateralismus - Zwischen Machtiibernahme und Parallelstruktur}

Im zweiten Workshop mit dem Titel Autokratien und Multilateralismus - Zwischen Machtübernahme und Parallelstruktur wurde unter der Leitung von Dr. Siegfried Schieder (Ruprecht-Karls-Universität Heidelberg) beleuchtet, welche Bedeutung Autokratien im multilateralen Kontext haben und wie sie in diesem agieren. Das Ziel des Workshops war, eine grundlegende Definition von Multilateralismus, die unterschiedlichen Typologien von Autokratien und das Konzept der internationalen Ordnung zu erlernen bzw. zu erfassen. Nach einer theoretischen Einführung zu den zentralen Konzepten Multilateralismus, Autokratie und internationale Ordnung sollte in Kleingruppen erarbeitet werden, inwieweit sich die zunehmende Autokratisierung von Demokratien vor allem seit den 1990er Jahren auf die multilaterale internationale Ordnung auswirkte und welche zentralen Unterschiede im 19., 20. und 21. Jahrhundert bestanden.

Anschließend wurde die Rolle von Autokratien in den internationalen funktionalen bzw. regionalen Teilordnungen thematisiert und in Anlehnung an die Forschung von Herrn Dr. Schieder die Positionierung von Autokratien in der internationalen Entwicklungszusammenarbeit vorgestellt. Entsprechend der Fragestellung, ob und inwieweit sich Autokratien und Demokratien bei der Vergabe von Entwicklungshilfe unterscheiden, konnte festgestellt werden, dass das Entwicklungsniveau (Human Development Index) einen wichtigeren Indikator für die Allokation von Entwicklungshilfe darstellt als der Regimetyp. Anknüpfend an dieses Ergebnis, das die Vorstellung einer klaren Dichotomie zwischen Autokratien und Demokratien (zumindest) im Bereich der Entwicklungshilfe infrage stellt, wurde diskutiert, ob diese aber in anderen Bereichen (z. B. Cyberraum) haltbar ist. In diesem Zusammenhang wurde auch darüber nachgedacht, die Vorstellung einer Dichotomie zwischen Demokratien und Autokratien insgesamt differenzierter zu beleuchten. Dabei wurde auch die Möglichkeit einer zunehmenden Lernfähigkeit autokratischer Staaten erörtert.

Zuletzt wurde in Bezug auf Autokratien und regionale sowie internationale Teilordnungen thematisiert, dass seit 1945 eine Ausbreitung von Regionalorganisationen zu beobachten ist. Während ein Beispiel der demokratischen Regionalisierung der Zusammenschluss europäischer Staaten in der EU darstellt, steht im autokratischen 
Regionalismus $^{1}$ die Verteilung von Ressourcen und Legitimation im Vordergrund. Im Rahmen der Besprechung des Konzepts der internationalen Ordnung und der funktionalen Teilordnungen fiel auf, dass das Verhalten von Demokratien und Autokratien gegenüber dem Multilateralismus nicht zwangsläufig so unterschiedlich ist, wie anfangs vermutet. Als Ergebnis des Workshops konnte damit festgehalten werden, dass die Analyse des Verhaltens von Autokratien im multilateralen Kontext ein höchst spannendes, aber auch umstrittenes Forschungsthema ist. Zukunftsprognosen seien durch eine disparate Forschung weiterhin problematisch.

Funding Open Access funding provided by Projekt DEAL.

Open Access Dieser Artikel wird unter der Creative Commons Namensnennung 4.0 International Lizenz veröffentlicht, welche die Nutzung, Vervielfältigung, Bearbeitung, Verbreitung und Wiedergabe in jeglichem Medium und Format erlaubt, sofern Sie den/die ursprünglichen Autor(en) und die Quelle ordnungsgemäß nennen, einen Link zur Creative Commons Lizenz beifügen und angeben, ob Änderungen vorgenommen wurden.

Die in diesem Artikel enthaltenen Bilder und sonstiges Drittmaterial unterliegen ebenfalls der genannten Creative Commons Lizenz, sofern sich aus der Abbildungslegende nichts anderes ergibt. Sofern das betreffende Material nicht unter der genannten Creative Commons Lizenz steht und die betreffende Handlung nicht nach gesetzlichen Vorschriften erlaubt ist, ist für die oben aufgeführten Weiterverwendungen des Materials die Einwilligung des jeweiligen Rechteinhabers einzuholen.

Weitere Details zur Lizenz entnehmen Sie bitte der Lizenzinformation auf http://creativecommons.org/ licenses/by/4.0/deed.de.

\footnotetext{
1 Obydenkova, A., \& Libman, A. (2019). Authoritarian regionalism in the world of international organi-
} zations. Global perspectives and the Eurasian enigma. Oxford: Oxford University Press. 